\title{
Evaluation of Urea Adsorption by Various Nanoporous Biomaterials
}

\author{
Wee-Keat Cheah and Fei-Yee Yeoh* \\ School of Materials and Mineral Resources Engineering, Universiti Sains Malaysia Engineering Campus, Malaysia
}

Received: June 5, 2018; Published: June 19, 2018

*Corresponding author: Fei -Yee Yeoh, School of Materials and Mineral Resources Engineering, Universiti Sains Malaysia Engineering Campus, 14300 Nibong Tebal, Penang, Malaysia

\begin{abstract}
High adsorption capacity and bio-friendly adsorbents are essential components to achieve miniaturization and improved portability while maintaining effective uremic toxin removal. Urea is targeted for removal mainly because it is the largest uremic toxin constituent. This paper evaluates the urea adsorption capacity of nanoporous materials activated carbon, activated carbon fiber and surface functionalized mesoporous silica. Results indicate that amine functionalized silica yielded the highest urea adsorption capacity through chemisorption interaction, despite possessing lower surface area. Sulfuric acid treated activated carbon fiber produced urea adsorption capacity close to that of amine functionalized mesoporous silica due to the high surface area generally through physisorption mechanism.
\end{abstract}

Keywords: Biomaterials; Porous Materials; Wearable Artificial Kidney; Activated Carbon Fiber; Mesoporous Silica; Renal Toxin

Abbreviations: WAK: Wearable Artificial Kidney; MS: Mesoporous Silica; ACF: Activated Carbon Fiber; EFB: Empty Fruit Bunch; CAC: Commercial Granular Activated Carbon; SG: Silica Gel; CS: Commercial Silica

\section{Introduction}

Wearable artificial kidney (WAK) typically employs activated carbon, urease and zirconium for the adsorption, decomposition and removal of urea and other uremic toxins from spent dialysate [1]. Adsorbents such as activated carbon is crucial for the regeneration of spent dialysate. This key system regenerates dialysate in a closed system, minimizing the need of fresh dialysate fluid supply. Activated carbon is a universal adsorbent for a majority of uremic toxins with the exception of urea [2]. Thus, urease is applied to catalyze the breakdown of urea into $\mathrm{NH} 3$ and $\mathrm{CO}_{2}$ [3]. The possible liberation of gases in the dialysate is a serious problem in the closed system dialysate. To mitigate such problem, nanoporous materials with strong affinity towards urea could be applied as urea adsorbents. This paper evaluates the urea adsorption capacity of two emerging nanoporous materials, i.e. mesoporous silica (MS) and activated carbon fiber (ACF).

Commercial silica and activated carbon are included as a benchmark for urea adsorption capacity evaluation. Urea is targeted in this study as it is the major uremic toxin constituent. The ease of surface functionalization of MS makes this material a selected candidate for a target specific adsorption [4]. Amine and diamine functionalization was selected to introduce seeding sites by inducing a urea crystallization-like effect [5]. ACF was selected because of its reportedly higher surface area compared to the granular or powdered variant [6]. The ACF was then treated with various organic and inorganic acids to improve surface hydrophilicity for better wetting to promote higher urea adsorption. The effect of chemisorption by surface modification and effect of physisorption by surface area on urea adsorption is compared to determine the predominant adsorption mechanism for better WAK adsorbent design.

\section{Materials and Methods}

Mesoporous silica SBA-15 was synthesized through a standard soft template route using Pluronics P123 (BASF) and tetraethyl orthosilicate (ACROS). The synthesized MS-S was subsequently functionalized with amine and diamine using (3-aminopropyl) triethoxysilane (Aldrich) and $\mathrm{N}$-(2-aminoethyl)-3-aminopropyltrimethoxysilane (Dow Corning) respectively. The synthesis process is similar as our previous report [7]. Activated carbon fiber was synthesized from oil palm empty fruit bunch (EFB) (United Palm Oil Mill, Nibong Tebal, Malaysia). The precursor was washed and subsequently treated with inorganic sulfuric acid (3M). Different acid treatments were repeated using inorganic acids nitic and phosphoric acid, as well as organic acids acetic and citric acid, with all acid concentration standardized at 3M. The treated precursor was 
then carbonized and activated in the same conditions similar as our previous report [8]. Aside from the synthesized ACF, MS and HAp samples, four other materials were included for urea adsorption capacity test. Commercial granular activated carbon (CAC) was added as a benchmark control sample.

Table 1: Matrix of samples for urea adsorption capacity test.

\begin{tabular}{|c|c|c|}
\hline Symbol & Sample Denotation & Material Description \\
\hline $\boldsymbol{\nabla}$ & IACF-S & Inorganic sulfuric acid activated ACF \\
\hline $\boldsymbol{\Delta}$ & IACF-N & Inorganic nitric acid activated ACF \\
\hline $\boldsymbol{\Delta}$ & IACF-P & $\begin{array}{c}\text { Inorganic phosphoric acid activated } \\
\text { ACF }\end{array}$ \\
\hline $\boldsymbol{\theta}$ & OACF-A & Organic acetic acid activated ACF \\
\hline $\boldsymbol{\theta}$ & OACF-C & Organic citric acid activated ACF \\
\hline $\boldsymbol{D}$ & CAC & Commercial activated carbon \\
\hline$\nabla$ & MS-S & Mesoporous silica SBA-15 \\
\hline$\theta$ & MS-N & Amine functionalized MS SBA-15 \\
\hline$\theta$ & MS-DN & Diamine functionalized MS SBA-15 \\
\hline
\end{tabular}

The adsorbent used by present artificial kidney prototypes are mainly granular activated carbon similar to the obtained CAC. Silica gel (SG) (Bendosen) was also included for this test as a benchmark for synthesized MS samples. The main difference between MS and silica gel lies in the respective pore size distribution. The pore size of MS could be controlled precisely to obtain a narrow distribution, which is desirable for target specific adsorption. Both silica gel and activated carbon are conventional adsorbents materials, with the latter applied commonly for water molecule adsorption as desiccants. The remaining material is bulk (non-porous) commercial silica (CS) (Fluka), as a control for surface chemistry study by eliminating pore effect. Table 1 shows the matrix of materials subjected to urea adsorption capacity test and their respective descriptions. Using the Beer-Lambert's law, a urea calibration curve was prior obtained using a set of standard urea solutions of known concentrations plotted against the absorption intensity obtained from UVVis spectrometer (Varians Cary 50) at low speed scanning mode. Intensity of absorption at urea characteristic peak $200 \mathrm{~nm}$ was used to plot the calibration curve for concentration of urea solutions. The 11 nanoporous and bulk samples were then subjected to urea adsorption test.

\section{Results and Discussion}

Figure 1 shows the specific adsorption capacity and surface area of various samples for the urea adsorption capacity test. The samples are arranged in the descending order of specific urea adsorbed amount. From a quick glance of this figure, there are two distinctive segregated groups; ACF group with higher surface and MS group with comparatively lower surface area. This initial evaluation showed that MS-N and IACF-S are the best candidate nanoporous biomaterials for urea adsorption, approximately $40 \%$ higher than the benchmark CAC. Although functionalization of MS-N from MS-S led to loss of surface area, the substitute amine functional groups improved the overall urea adsorption capacity by $\sim 10 \%$. CS possesses the lowest urea adsorption capacity because of the non-porous structure despite possessing similar surface chemistry as compared to higher adsorption performance MS-S and SG. IACF-S performed better than the benchmark CAC, an indication that palm oil EFB are promising AC precursor particularly when treated with sulfuric acid. Further surface chemistry analysis using the Fourier transform infrared spectroscopy revealed that IACF-S possesses the highest qualitative amount (peak intensity) of carbonyl group among all ACF samples.

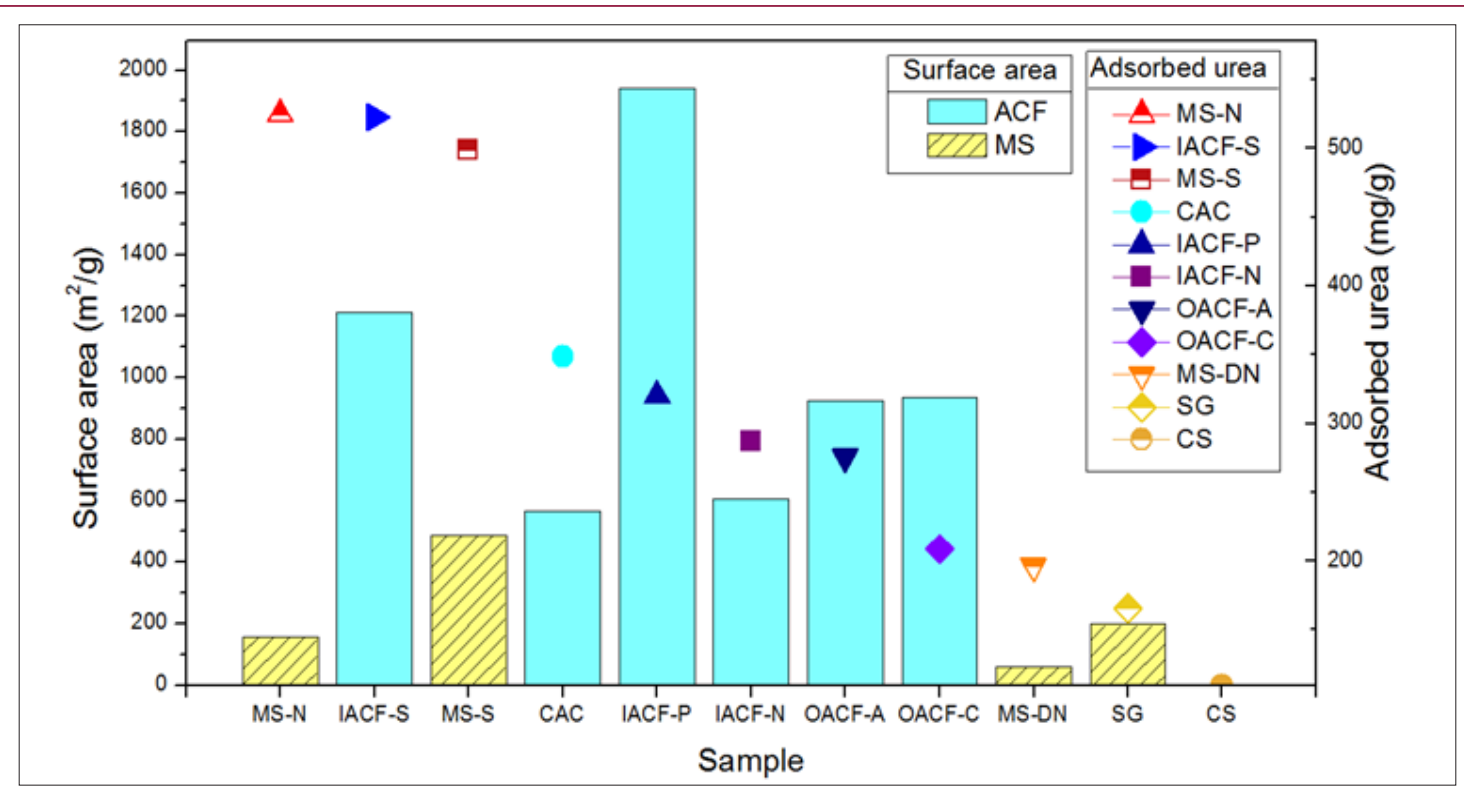

Figure 1: Surface area and adsorption capacity of nanoporous biomaterials and commercial samples. 
The active carboxyl group is effective in increasing the affinity of ACF towards the adsorption of urea. Hydrogen bonding between the carboxyl groups with the amine functional group of urea is much stronger than the weak van der Waals forces formed during the adsorption of urea on the hydrocarbon surface. Given the right synthesis conditions, EFB derived ACF could be used to replace GAC, specifically for urea adsorption. The urea adsorption capacity of IACF-P is lower than both IACF-S and CAC despite possessing a higher surface area. Phosphoric acid is commonly used for acid activation and causes severe devolatilization on the organic surface, effectively removes active functional groups from the organic surface at the expense of higher surface area. The possibility of utilizing organic acids for activation is of particular interest in this project due to the possible contamination of dialysate fluid by residual inorganic acid. The urea adsorption capacity of OACF-A $(276.6 \mathrm{mg} / \mathrm{g})$ is slightly lower than that of CAC $(348.8 \mathrm{mg} / \mathrm{g})$.

Given that acetic acid is an organic acid, subsequent studies to improve uremic toxin adsorption properties using this acid for activation process could be employed moving forward from this preliminary study. To further understand the relationship between urea adsorption capacity, the normalized surface area adsorption capacity, i.e. the number of molecules adsorbed per unit area (M/a), is tabulated. Figure 2 shows the M/a for ACF and MS. The M/a for MS-N and MS-DN are magnitudes higher pared to the remaining samples. Typically, pure physisorption involves multilayer adsorption of approximately 3 layers. Urea adsorption for CAC and ACF samples are thus confirmed to be of physisorption in nature. Urea adsorption by SG and MS-S show signs of favorable surface chemistry, since both these samples are silica $\left(\mathrm{SiO}_{2}\right)$. The surface functionalization of amine and diamine groups for MS-N and MS-DN resulted in chemisorption type adsorption. Though diamine shows strong chemical interactions with urea, the low MS-DN surface area nevertheless reduced the overall urea adsorption capacity. On a separate note, the M/a for non-porous CS was excluded due to low accuracy and large measurement error because of the extremely low surface area, thus it is not included in Figure 2. Since the surface chemistry of CS is similar to that of MS-S and SG, the expected M/a for CS is approximately 10.

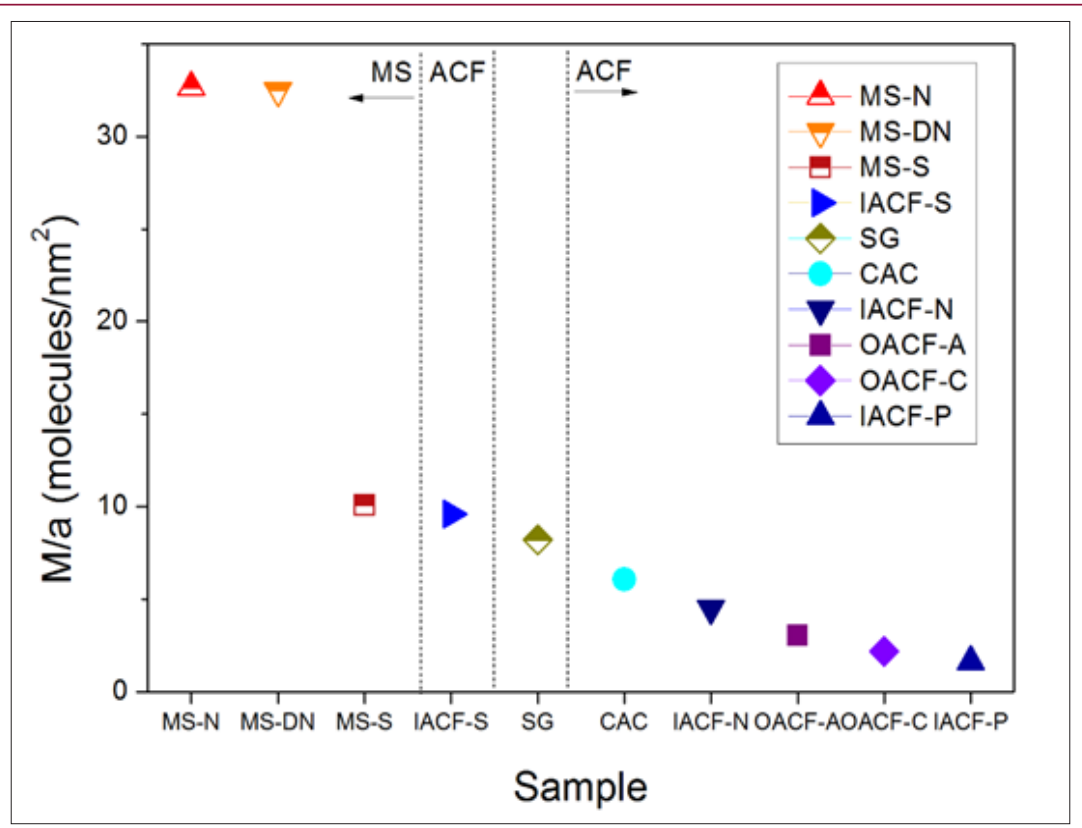

Figure 2: Normalized surface area adsorption capacity (M/a) for ACF and MS.

\section{Conclusion}

Based on the urea adsorption evaluation, amine functionalized mesoporous silica and sulfuric acid treated activated carbon fiber could potentially be applied as excellent nanoporous adsorbent materials for dialysate regeneration in a wearable artificial kidney. Both materials are applicable due to 2 distinctive concepts: amine MS functionalization facilitated the adsorption of multiple urea layers through chemisorption while IACF-S exhibited a high surface area, which allows for more physiosorbed urea loading. Good urea adsorption was found to rely on not either one particularly good surface area or surface chemistry, but a combination of both factors. Application of such target specific and high surface nanoporous materials would enable further miniaturization of the present wearable artificial kidney.

\section{Acknowledgement}

Authors would like to thank the Ministry of Education Malaysia, Ministry of Science, Technology and Innovation Malaysia (MOSTI), My Brain15 and AUN/SEED-Net for the financial support provided.

\section{References}

1. Gura V, Rambod E (2010) Dual-ventricle pump cartridge, pump, and method of use in a wearable continuous renal replacement therapy device. Google Patents.

2. Drukker W, Parsons FM, Maher JF (2012) Replacement of Renal Function by Dialysis: A textbook of dialysis. In Drukker W, F M Parsons, JF Maher (Eds.). Springer Science \& Business Media, Berlin, Germany.

3. Hojjatie M, John Clapp, Thomas Fairweather, Constance Lockhart (2009) Calcium polysulfide, potassium polysulfide, calcium thiosulfate, and magnesium thiosulfate as urease inhibitors. Google Patents. 
4. Fine D, Grattoni A, Goodall R, Bansal SS, Chiappini C, et al. (2013) Silicon micro-and nanofabrication for medicine. Adv Healthc Mater 2(5): 632-666.

5. Godfrey PD, Brown RD, Hunter AN (1997) The shape of urea. J Mol Struct 413: 405-414.

6. Yang K, Peng J, Srinivasakannan C, Zhang L, Xia H, et al. (2010) Preparation of high surface area activated carbon from coconut shells using microwave heating. Bioresource technol 101(15): 6163-6169.
7. Cheah WK, Thung WL, Othman R, Yeoh FY (2013) Preparation and Characterization of Amine-Functionalised SBA-15. Adv Mater Res 686: 336-350.

8. Cheah WK, Othman R, Yeoh FY (2014) Organic and Inorganic Acid Activation of Activated Carbon Fiber from Palm Oil Empty Fruit Bunch. Adv Mater Res 858: 122-130.
(C) Co This work is licensed under Creative

Submission Link: https://biomedres.us/submit-manuscript.php

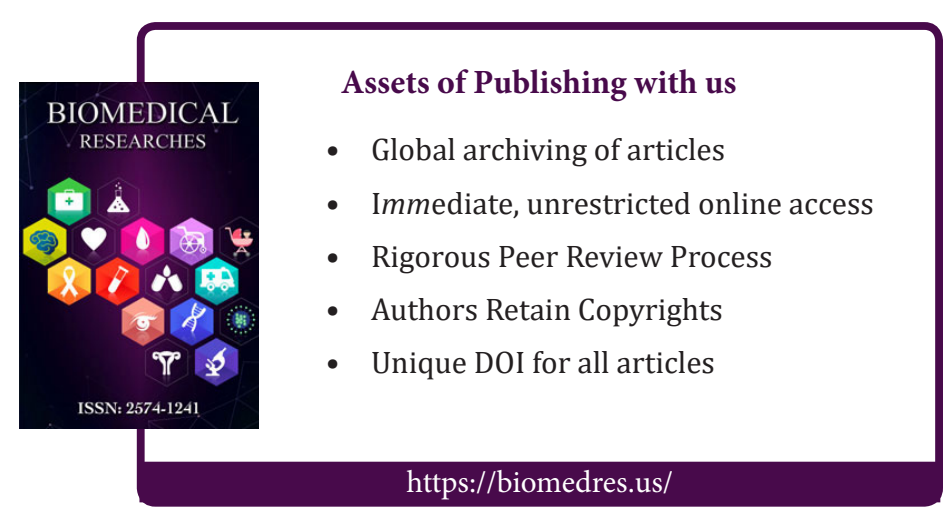

